\title{
DNA walker circuits: computational potential, design, and verification
}

\author{
Frits Dannenberg ${ }^{1}$, Marta Kwiatkowska ${ }^{1}$, \\ Chris Thachuk ${ }^{1}$, and Andrew J. Turberfield ${ }^{2}$ \\ 1 University of Oxford, Department of Computer Science, Wolfson Building, Parks Road, \\ Oxford, OX1 3QD, UK \\ ${ }^{2}$ University of Oxford, Department of Physics, Clarendon Laboratory, Parks Road, Oxford, \\ OX1 3PU, UK
}

\begin{abstract}
Unlike their traditional, silicon counterparts, DNA computers have natural interfaces with both chemical and biological systems. These can be used for a number of applications, including the precise arrangement of matter at the nanoscale and the creation of smart biosensors. Like silicon circuits, DNA strand displacement systems (DSD) can evaluate non-trivial functions. However, these systems can be slow and are susceptible to errors. It has been suggested that localised hybridization reactions could overcome some of these challenges. Localised reactions occur in DNA 'walker' systems which were recently shown to be capable of navigating a programmable track tethered to an origami tile. We investigate the computational potential of these systems for evaluating Boolean functions. DNA walkers, like DSDs, are also susceptible to errors. We develop a discrete stochastic model of DNA walker 'circuits' based on experimental data, and demonstrate the merit of using probabilistic model checking techniques to analyse their reliability, performance and correctness.
\end{abstract}

\section{Introduction}

The development of simple biomolecular computers is attractive for engineering and health applications that require in vitro or in vivo information processing capabilities. DNA computing models which use hybridization and strand displacement reactions to perform computation have been particularly successful. DNA strand displacement systems (DSD) have been shown experimentally to simulate logic circuits $[13,12]$ and are known to be Turing-universal [11]. However, computing with biomolecules creates many challenges. For example, reactions within a DSD are global in the following sense: strands which are intended to react must first encounter one another in a mixed solution. The mixing of all reactants may lead to unintended reactions between strands. These systems do not, at present, ensure the spatial locality typical of other computing models. Qian and Winfree suggested that tethering DNA based circuits to an origami tile could overcome some of these challenges [12]. This idea was explored and expanded upon by Chandran et al. [5], who investigate how such systems could be realised experimentally, give constructions of composable circuits, and propose a biophysical model for verification of tethered, hybridization-based circuits. Our work is largely inspired 
Stepping mechanics

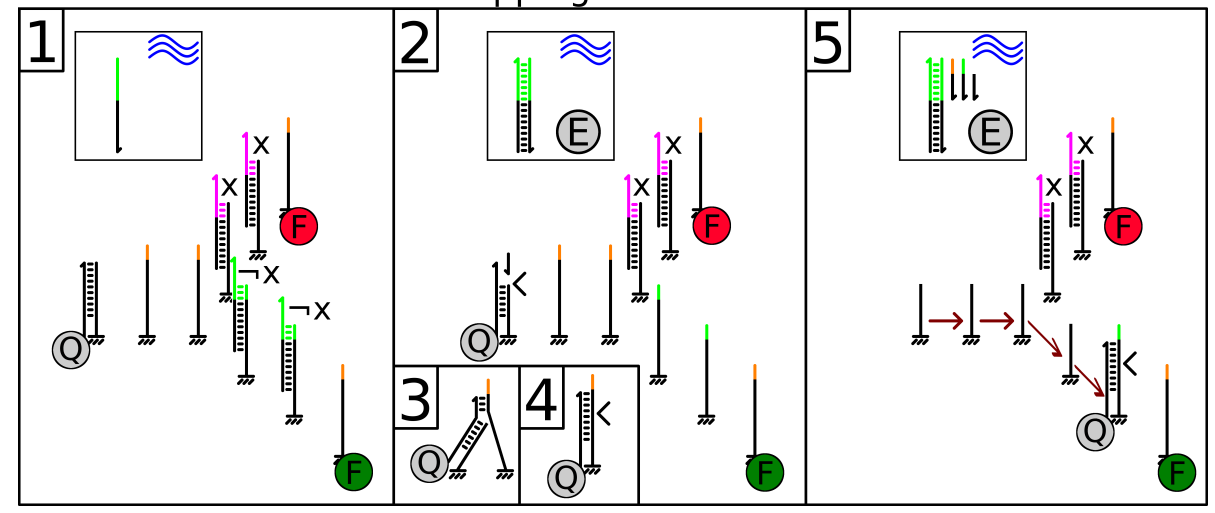

Fig. 1. (1) The walker strand carries a load (Q) that will quench fluorophores (F) when nearby. The walker is attached to the initial anchorage and all other anchorages are blocked. By adding unblocking strands, the selected track becomes unblocked. In this case the signal that opens up the path labelled by $\neg X$ is added. (2) The nicking enzyme (E) attaches to the walker-anchorage complex, and cuts the anchorage. The anchorage top melts away from the walker, exposing 6 nucleotides as a toehold. (3) The exposed toehold becomes attached to the next anchorage. (4) In a displacement reaction, the walker becomes completely attached to the new anchorage. The stepping is energetically favourable, because it re-forms the base pairs that were lost after the previous anchorage was cut. (5) Repeating this process, the walker arrives at a junction. The walker continues down the unblocked track, eventually reaching the final anchorage and quenching the fluorophore.

by theirs, but we consider another setting which also exhibits localised reactions: DNA walker systems $[2,7,10,14-16]$.

Various DNA walkers have been experimentally realised — see [14] and references therein. Single-legged DNA walkers were recently shown capable of navigating a programmable track of strands, called anchorages, that are tethered to a DNA origami tile [14]. Movement of the walker between anchorages is shown in Fig. 1. Initially, all tracks are blocked by hybridization to blocker strands. Anchorages and their blockers are addressed by means of distinct toehold sequences (shown coloured): anchorages are selectively unblocked by adding strands complementary to their blockers as input. Much like field programmable gate arrays, these systems are easily reconfigured. By using programmable anchorages at track junctions, Wickham et al. [14] demonstrate that a walker can be directed to any leaf in a complete two-level binary tree using input strands that unblock the intended path.

In Section 2, the computational expressiveness of such walker systems is explored, using a theoretical framework that assumes ideal conditions. We highlight significant limitations of current walker systems and motivate future work. In Section 3 we develop a probabilistic model to analyse the impact of different sources of error that arise in experiments on reliability, performance and correctness of the computation. The model can be used to support the design and verification of DNA walker circuits. 


\section{Computational potential of DNA walker circuits}

In this section we explore the computational potential of DNA walker systems. We focus on deterministic Boolean function evaluation and call the resulting constructions DNA walker circuits. We begin by defining a model of computation that makes explicit the underlying assumptions that characterize the DNA walker systems considered here. These assumptions are consistent with current published experimental systems: in particular, we do not explore the potential for multiple walkers to interact within the same circuit. However, we do consider the potential consequences for parallel computation.

\subsection{A model of computation for DNA walker circuits}

A DNA walker circuit is composed of straight, undirected, tracks (consecutive anchorages), and gates (track junction points) that connect at most three tracks. A gate can have at most one Boolean guard for each track that it connects. A particular guard is implemented using one or more blocking strands that share a common toehold sequence; distinct guards use distinct toehold sequences. A track adjacent to a gate is blocked if it has a guard that evaluates to false - its unblocking strands are not added to solution - and is unblocked otherwise. For example, Fig. 1 depicts a circuit of a single gate connecting three tracks. The track ending with the anchorage marked with the red fluorophore (top right of panel 1) has the Boolean guard $X$, while the track ending with the anchorage marked with the green fluorophore has the Boolean guard $\neg X$. Panel 2 of Fig. 1 shows that the path to the green fluorophore is unblocked when $\neg X$ evaluates to true (i.e., the unblocking strands for $\neg X$ are added to solution). In this case, $X$ evaluates to false and the path to the red fluorophore remains blocked (i.e., the unblocking strands for $X$ are not added to solution). We define a fork gate as having at most one input track, and exactly two guarded output tracks. Each circuit has one source - a fork gate with no input track denoting the initial position of a walker. A join gate with an output track has at most two guarded input tracks. A join gate with no output track is a sink and has at most three (unguarded) input tracks. Each circuit has one or more true sinks and one or more false sinks.

In a circuit $\mathcal{C}$ with Boolean guards over $n$ variables, a variable assignment $A$ for $\mathcal{C}$ is a truth assignment of those $n$ variables. Consider any DNA walker circuit $\mathcal{C}$ and variable assignment $A$ for $\mathcal{C}$. Let $\mathcal{C}[A]$ denote the set of reachable paths originating from the source of $\mathcal{C}$, after all guards are evaluated as blocked or unblocked, under assignment $A$. We say that $\mathcal{C}$ is deterministic under assignment $A$ if there is exactly one path from the source to a $\operatorname{sink}$ in $\mathcal{C}[A]$. Note that this definition of determinism precludes the possibility of a deadlock, (i.e., when no path from the source can reach a sink). Let VALUE $(\mathcal{C}[A])$ be the output value of the circuit under assignment $A$ (i.e., whether the reachable sink is a true sink or a false $\operatorname{sink}$ ). Circuit $\mathcal{C}$ is deterministic if it is deterministic under all possible variable assignments.

A circuit set $\mathrm{S}$, consisting of one or more unconnected circuits, is deterministic if and only if VALUE $\left(\mathcal{C}_{i}[A]\right)=\operatorname{VALUE}\left(\mathcal{C}_{j}[A]\right)$, for each $\mathcal{C}_{i}, \mathcal{C}_{j} \in \mathrm{S}$, under any possible assignment $A$. Let VALUE $(\mathrm{S}[A])$ be the value of $\mathrm{S}$ under assignment $A$. The size of $\mathrm{S}$, denoted by SIZE (S), is the total count of component gates. ${ }^{3}$ We define the worst

\footnotetext{
${ }^{3}$ We do not investigate circuit area in this paper.
} 
case time of a computation in S, denoted by $\operatorname{TIME}(\mathrm{S})$, as the longest reachable path from a source to a sink. This notion of time captures the ability of multiple walkers to simultaneously traverse disjoint paths (one per unconnected circuit).

Let $\mathrm{S}[A]$ denote the set of reachable paths in $\mathrm{S}$ under assignment $A$ (one per unconnected circuit). Given a circuit $\mathcal{C}_{i} \in \mathrm{S}$, we say that a gate $G \in \mathcal{C}_{i}$ is reachable in $\mathrm{C}_{i}[A]$ (equivalently $\mathrm{S}[A]$ ) if there exists an unblocked path from the source of $\mathcal{C}_{i}$ to $G$. Note that, if every gate is reachable, this implies that every output track of a gate can be traversed under some variable assignment. We call gates where this is not true redundant. We will reason about circuit sets where all gates are reachable and non-redundant under some variable assignment. When this is not the case, the circuit set can be simplified to one that is logically equivalent.

\subsection{Reporting output in DNA walker circuits}

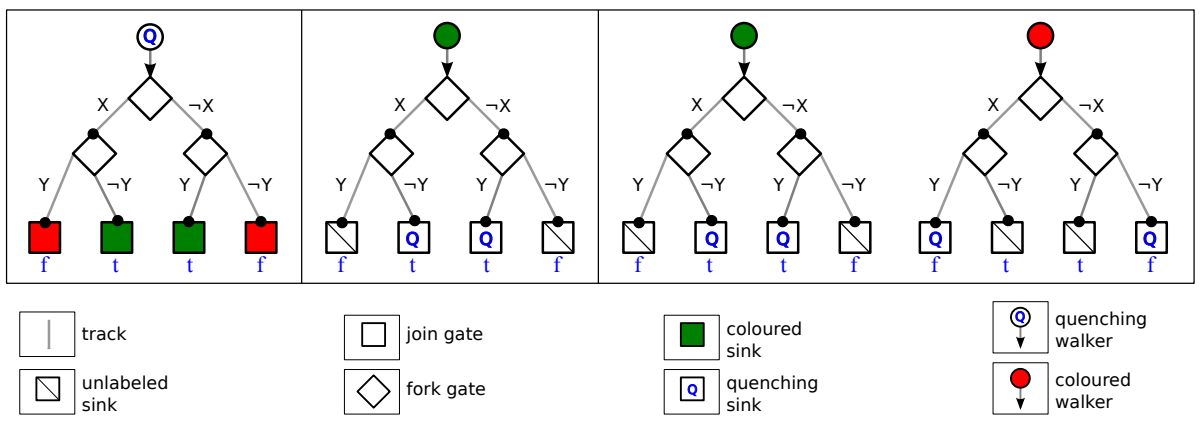

Fig. 2. Reporting Boolean decisions with DNA walker circuits. (Left) A quenching walker with red fluorophores labelling false sinks and green fluorophores labelling true sinks. A drop in signal for one colour indicates the truth value of the circuit. However, the signal drop is inversely proportional to the number of sinks of the same colour. (Center) A green coloured walker and quenching true sinks. When the circuit evaluates to true the green signal is fully suppressed. However, the fluorescence output from this circuit cannot distinguish between an incomplete computation and a false one. (Right) Two parallel copies of the circuit, with different fluorophores labelling the walkers and with quenching true sinks in one and quenching false sinks in the other: the computation is complete and unambiguously reported when one colour is suppressed.

Output of a DNA walker circuit can be reported with the use of different coloured (spectrally resolvable) fluorophores and also quenchers. If a walker carries a quencher cargo, then it has the potential to decrease one of a number of different fluorescent signals from fluorophores positioned at the circuit sinks. This scenario is illustrated in Fig. 2 (Left). In a circuit that decides a Boolean function, a single, quenching, walker can only decrease the signal of a particular colour (corresponding to a particular fluorophore) by an amount that is inversely proportional to the number of sinks labelled with that same colour. Accurate output reporting could be problematic in larger circuits 
with many sinks. We will therefore focus only on reporting strategies that fully suppress a particular colour. Rather than carrying a quencher, a walker instead carries a fluorophore of a single colour and either all true sinks or all false sinks are labelled with quenchers. An example with quenching true sinks is shown in Fig. 2 (Center). This circuit can fully suppress the fluorophore signal when it evaluates to true, regardless of its size. However, this is a one-sided reporting strategy as one cannot distinguish between the case of an incomplete computation or one evaluating to false. As illustrated in Fig. 2 (Right) this shortcoming can be addressed by using two circuits in parallel with each using a one-sided reporting strategy. Each of the two (otherwise identical) circuits uses a different coloured walker: one has quenching false sinks and the other quenching true sinks. In this circuit set, one colour will be fully suppressed when it is true, the other when it is false, and neither will be suppressed until the computation completes.

\subsection{Deterministic fork and join gates in DNA walker circuits}

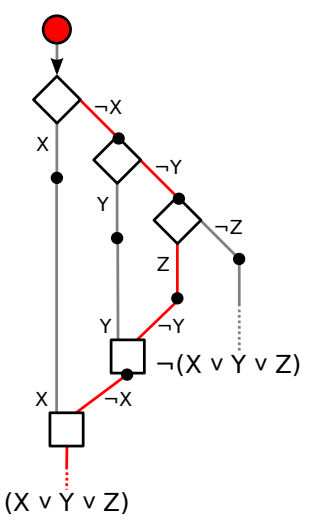

(a)

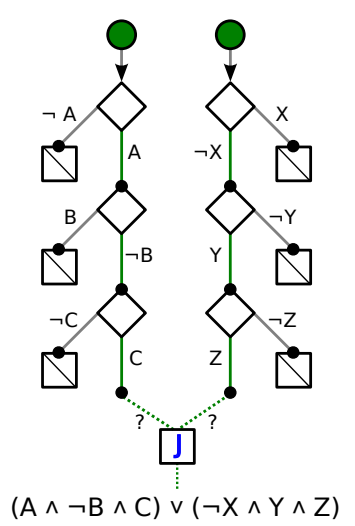

(b)

Fig. 3. (a) A connectivity graph of a DNA walker circuit to evaluate the disjunction $(X \vee Y \vee Z)$. There are two output tracks: one when the circuit evaluates to true, the other when it evaluates to false. The resulting path when $X=Y=f$ and $Z=t$ is shown highlighted. (b) Two conjunction circuits are composed into the disjunction $(A \wedge \neg B \wedge C) \vee(\neg X \wedge Y \wedge Z)$. Two source nodes (two walkers) are used to evaluate clauses in parallel. No assignment of guards to the join gate labelled $J$ can ensure that this circuit is deterministic. This is evident when $A=C=Y=Z=t$ and $B=X=f$.

If all gates in a circuit set $S$ are deterministic, it follows that $S$ is deterministic. The following theorem shows that deterministic fork gates must have output guards that are negations of each other.

Theorem 1. A fork gate in a DNA walker circuit is deterministic if and only if there exists some guard $G$ such that the left output track is guarded by $G$ and the right is guarded by $\neg G$. 
Proof. If neither output track is guarded, then any path that can reach the gate could be extended via the left or the right output track and the gate would not be deterministic. Similarly, this is true when only one output track is guarded and the guard evaluates to false. (If the fork gate is only reachable when the single output guard evaluates to true, then the gate is redundant as the output track with the guard is never used.) Thus, consider when each output track is guarded and let the left output have guard $G_{L}$ and the right have guard $G_{R}$. Note that $G_{L} \not \equiv G_{R}$ as otherwise any path that reaches the gate will result either in a deadlock — when both evaluate to false — or the path could be extended via the left or the right output tracks - when both evaluate to true. Consider any path $p$ that can reach the gate and the case when $G_{L}$ evaluates to true and $G_{R}$ to false. It follows that, before reaching the gate, $p$ must not traverse a track guarded by $\neg G_{L}$ nor by $G_{R}$. Since the gate is non-redundant, $p$ must also be able to reach the gate when $G_{L}$ evaluates to false and $G_{R}$ to true. It follows that, before reaching the gate, $p$ must not traverse a track guarded by $G_{L}$ nor by $\neg G_{R}$. Therefore, path $p$ is independent of the variables affecting guards $G_{L}$ and $G_{R}$. Thus, there exists a variable assignment such that any path reaching the gate will result in a deadlock, or can be extended via both output tracks, unless $G_{L} \equiv \neg G_{R}$.

Given any Boolean function $f:\{0,1\}^{n} \rightarrow\{0,1\}$, there exists a deterministic DNA walker circuit set $\mathrm{S}$ that can evaluate $f$, under any assignment to its $n$ variables, such that TIME $(\mathrm{S})=O(n)$. One construction is to simply form a canonical binary decision tree over some fixed order of the $n$ variables. However, in such a construction SIZE $(\mathrm{S})=\Theta\left(2^{n}\right)$. It is natural to consider more space efficient representations to evaluate $f$, such as binary decision diagrams (BDDs) [4]. In particular, reduced ordered BDDs are capable of representing some Boolean functions in a compressed form that can be exponentially smaller than its canonical binary decision tree representation. Like walker circuits, BDDs have a unique source. Unlike general BDDs, DNA walker circuits are necessarily planar. Either we are limited to considering planar BDD representations or additional fork and join nodes must be added to a BDD representation when realising it as a walker circuit. A significant difference, however, is that BDDs form directed acyclic graphs while tracks in a DNA walker circuit are undirected. Consider the case when a walker reaches a join gate via its left input track. Unless the right input track is blocked, the walker is equally likely to continue on the right input track as it is on the output track. Additional steps are necessary to compensate for the undirected nature of tracks in walker circuits.

Unlike fork gates, it is not obvious whether all join gates can be made deterministic. Theorem 2 characterizes both the necessary and sufficient conditions: a deterministic join of two disjoint sets of paths, one for each input track, is only possible if they were previously "forked" 4 on some variable $X$ (i.e., in one set all paths traverse an edge guarded by $X$ and in the other set all traverse an edge guarded by $\neg X$ ). This property is exemplified by the contrast between the disjunction circuit of Fig. 3(a) and the disjunction of two conjunctions circuit as shown in Fig. 3(b). In the latter, two walkers are used in an attempt to parallelize the evaluation. However, as the clauses do not have literals over a common variable, there are no guards that can be assigned to

\footnotetext{
${ }^{4}$ It is not a necessary condition that the two disjoint sets of paths reaching the join were forked by a common gate, only that they can be partitioned based on the value of some variable.
} 
the join gate labeled $J$ to ensure the circuit is deterministic. Note that this limitation is not caused by the restricted topology of walker circuits (i.e., their layout on a planar surface), but rather by the property that their tracks are undirected.

Theorem 2. A join gate in a DNA walker circuit is deterministic if and only if there exists some guard $G$ such that the left input track is guarded by $G$, the right by $\neg G$ and, prior to reaching those guards, all paths that can reach the left input must traverse a track guarded by $G$ and all paths that can reach the right must traverse a track guarded by $\neg G$.

Proof. ( $\Rightarrow$ if) Suppose the left input track is guarded by $G$, the right by $\neg G$ and, prior to reaching those guards, all paths that can reach the left input must traverse a track guarded by $G$ and all paths that can reach the right must traverse a track guarded by $\neg G$. There are two cases to consider. Suppose $G$ evaluates to true. Then, no path can reach the right input since, by the assumption, those paths must traverse a track guarded by $\neg G$ prior to reaching the gate. It follows that all paths that can reach the gate when $G$ evaluates to true must be to the left input. Furthermore, as the right input is guarded by $\neg G$, those paths can only be extended via the output of the gate. The other case ( $G$ evaluates to false) is symmetric. Furthermore, as the guards are negations of each other, they cannot simultaneously evaluate to false and cause a potential deadlock.

( $\Leftarrow$ only if) Let $G_{L}$ and $G_{R}$ be the guards of the left and right inputs, respectively. (If one or more of the input tracks is unguarded, then the gate cannot be deterministic when both are reachable by at least one path.) First, consider all paths that can reach the left input, guarded by $G_{L}$. It must simultaneously be true that none of those paths (i) traverse a track guarded by $\neg G_{L}$ and (ii) all of those paths traverse a track guarded by $\neg G_{R}$. If condition (i) is not satisfied, then there would exist a path that traverses a track guarded by $\neg G_{L}$ and, to extend past the join gate, must traverse another guarded by $G_{L}$. As this is not possible, the path would end in a deadlock and the gate would not be deterministic. If condition (ii) is not satisfied then there would exist some path $p$ that does not traverse a track guarded by $\neg G_{R}$, but may possibly traverse a track guarded by $G_{R}$. In this case, there exists a variable assignment where $G_{R}$, and all other guards on path $p$, evaluate to true. With such a variable assignment, path $p$ could be extended via the output track or the right input track. Thus, condition (ii) must also be satisfied, as otherwise the gate would not be deterministic. The conditions (and the argument that both are necessary) when considering all paths that can initially reach the right input, guarded by $G_{R}$, are symmetric.

The sufficiency argument ( $\Rightarrow$ if) shows the gate is deterministic when $G_{L} \equiv \neg G_{R}$. It remains to show it is not deterministic otherwise. First, consider the consequence when both $G_{L}$ and $G_{R}$ evaluate to true. By condition (ii) all paths leading to the left (right) input traverse a track guarded by $\neg G_{R}\left(\neg G_{L}\right)$. In this case, no paths can reach the gate. Recall that the gate is non-trivial and therefore each input is reachable by at least one path. Thus, consider when both $G_{L}$ and $G_{R}$ evaluate to false. The conditions permit that paths can reach the gate; however, if any path does it will deadlock as both inputs to the gate are blocked. Thus, for all paths that can reach the gate, it will be deterministic only when $G_{L} \equiv \neg G_{R}$. 


\subsection{Evaluating Boolean formulas with DNA walker circuits}

Despite the shortcomings of join gates in current DNA walker circuits, it is not the case that Boolean formulas must be evaluated using a circuit forming a binary decision tree. Any Boolean formula can be represented in one of its canonical forms. We will focus on conjunctive normal form (CNF) which is a single conjunction of clauses, where each clause is a disjunction over literals. A formula in CNF is said to be $k$-CNF if the largest clause has size $k$. Using a standard transformation, a Boolean formula in $k$-CNF with at most $l$ total literals can be converted to a 3-CNF formula over $O(l)$ variables, with at most $O(l)$ clauses (each having at most 3 literals). As such, we will reason exclusively about circuits to evaluate $3-\mathrm{CNF}$ formulas.

Constructing a walker circuit to represent a formula in 3-CNF with $m$ clauses is straightforward. Each clause can be represented by the disjunction circuit of Fig. 3(a). The source of the circuit will be the first fork gate of the first clause. The output track signalling the $i$-th clause is satisfied is connected to the input track of clause $i+1$. Thus, the walker will only reach the single true sink of the circuit (output from clause $m$ ) if the formula is satisfied for that particular variable assignment. To ensure that both true and false signals can be reported deterministically, we use the reporting strategy depicted in Fig. 2 (Right) which employs two parallel copies of the circuit, each using different coloured walkers and different quenching sinks.

Theorem 3. Let $\mathcal{F}$ be any 3-CNF Boolean formula with $m$ clauses. There exists a DNA walker circuit set $\mathrm{S}$, with $\mathrm{SIZE}(\mathrm{S})=\Theta(m)$ and $\operatorname{TIME}(\mathrm{S})=O(m)$, such that given any variable assignment $A$ for $\mathcal{F}$, VALUE $(\mathrm{S}[A])$ is the truth value of $\mathcal{F}$ under assignment A.

Proof. The construction is described in Section 2.4 and it is easy to see that the circuit is deterministic and that it correctly reports the truth value of $\mathcal{F}$ under assignment $A$. What remains is to bound the circuit size and worst case time. The construction uses a set of two circuits: $\mathcal{C}_{T}$ and $\mathcal{C}_{F}$. Consider the circuit $\mathcal{C}_{T}$ used to evaluate if $\mathcal{F}$ is true under assignment $A$. There are $m$ clauses and each is simulated by a disjunction circuit of size $O(1)$. These circuits are composed in series to form $\mathcal{C}_{T}$. Therefore, $\operatorname{SIZE}\left(\mathcal{C}_{T}\right)=\Theta(m)$ and $\operatorname{TIME}\left(\mathcal{C}_{T}\right)=O(m)$. The arguments are the same for circuit $\mathcal{C}_{F}$ and, as both are evaluated in parallel, the claim follows.

While the construction of Theorem 3 can represent any Boolean formula, and some in exponentially less space than a binary decision tree, the resulting circuit set is formula specific. Given the effort of creating DNA walker circuits, a more uniform circuit — one capable of evaluating many Boolean functions - is worth exploring. As with silicon circuits, we can construct a uniform circuit to evaluate any 3-CNF formula, under any variable assignment, up to some bound on the number of variables. Each variable can be present in a clause as either a positive or negative literal, but not both. (The circuit can be modified to handle this case if necessary.) Therefore, there are at most $2^{3}\left(\begin{array}{l}n \\ 3\end{array}\right)$ unique clauses in any 3-CNF Boolean formula over $n$ variables, and also for any formula over $m \leq n$ variables. In this general circuit, we supplement each possible clause with an initial fork gate guarded on the condition of the clause being active or inactive in the particular formula being evaluated. If it is inactive, the walker can pass through to the 
output track denoting true, without traversing guards for the literals of the clause. Note that this only increases the size of each clause by a constant.

Corollary 1. There exists a DNA walker circuit set $\mathrm{S}$, with $\operatorname{SIZE}(\mathrm{S})=O\left(n^{3}\right)$ and $\operatorname{TIME}(\mathrm{S})=O\left(n^{3}\right)$, that can evaluate any $3-C N F$ Boolean formula over $m \leq n$ variables under any variable assignment.

A 3-CNF formula with $m$ clauses can be evaluated in polylogarithmic time (in $m$ ) using a silicon circuit in a straightforward manner: each clause can be evaluated in parallel and those results can be combined using a binary reduction tree of height $O(\log m)$-only if all clauses are satisfied will the root of the reduction tree output true. Is the same possible in DNA walker circuits? Unfortunately, this is not the case in general. Such a circuit would require a new kind of join gate, outside of our current model of computation, to perform a conjunction of multiple walkers - one walker leaves the gate only after all input walkers have arrived. Parallel evaluation of circuits representing formulas in disjunctive normal form (DNF) does not fair better. Consider the case of a DNF formula with $m$ clauses where clause $m-1$ and clause $m$ have no literals over a common variable. By Theorem 2, a join gate connecting the circuits for these clauses cannot be deterministic. An example of this situation is given in Fig. 3(b).

\section{Design and verification of DNA walker circuits}

We have so far assumed DNA walker circuits to work perfectly. In a real experiment various errors can occur, for example, the walker may release from the track, or a blockade can fail to block an anchorage. In this section, we analyse the reliability and performance of DNA walker circuits using probabilistic model checking. We develop a continuous-time Markov chain model, based on a variety of DNA walker experiments from $[2,14,15]$, and analyse it against quantitative properties such as the probability of the computation terminating or expected number of steps taken until termination. We use the PRISM model checker [8], which accepts models described in a scripted language and properties in the form of temporal logic. For example, if we label all states of the model where a walker quenches any fluorophore by "finished", then the query $P_{=?}\left[F^{[T, T]}\right.$ finished $]$ yields the probability of all paths that eventually reach a state where a walker has quenched a fluorophore (in other words, the computation terminated) by time $T$. A custom tool was developed to generate PRISM model scripts with matching track-layout graphs. Different configurations of tracks are studied: linear tracks are considered in Fig. 4 (Top), while branched tracks are used in Fig. 5 and Fig. 6. We use the results of experiments on linear (Fig. 4) and single-branched tracks to establish model parameters, and match model predictions with observations on double-layer tracks to evaluate the quality of our model.

Experiments show that the walker can step onto anchorages that are fixed as far away as $19 \mathrm{~nm}$. We assume non-zero rates for the walker to step onto any intact anchorage within $24 \mathrm{~nm}$ distance. This range was chosen by taking into account the lengths of the empty anchorage and walker-anchorage complex, estimated around $15 \mathrm{~nm}$ and 11 $\mathrm{nm}$ respectively. 

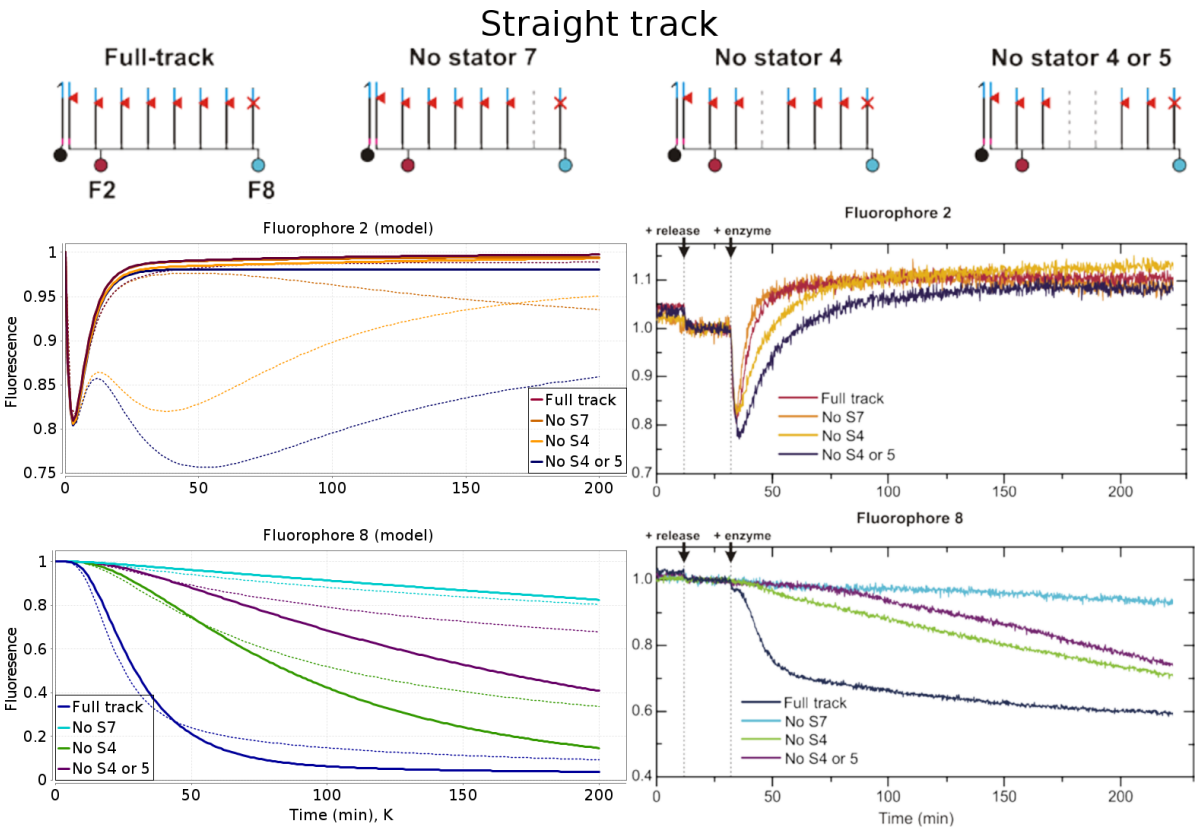

Fig. 4. Top: A small linear track of 8 anchorages with fluorophores on both the second and last anchorage. Experiments were performed with one or more anchorages omitted [15]. Right: Experimental results (reproduced with permission from the authors). The walker hardly reaches the final anchorage when anchorage 7 is removed, due to the double penalty of a longer final step and the mismatch in the final anchorage. Left: Model results. Dotted lines: Alternative model where the walker can step onto already-cut anchorages with rate $k_{b}=k / 30$.

A step taken by the walker corresponds to a single transition in the Markov chain, although the real stepping process is more complex, as depicted in Fig. 1. Assume that the stepping rate $k$ depends on distance $d$ between anchorages and some base stepping rate $k_{s}$. Denote by $d_{a}=6.2 \mathrm{~nm}$ the average distance between anchorages in the experiment shown in Fig. 4. Denote by $d_{M}=24 \mathrm{~nm}$ the maximal interaction distance discussed earlier. Based on previous experimental estimates of [15], we fix the stepping rate $k$ as:

$$
k= \begin{cases}k_{s}=0.009 \mathrm{~s}^{-1} & \text { when } d \leq 1.5 d_{a} \\ k_{s} / 50 & \text { when } 1.5 d_{a}<d \leq 2.5 d_{a} \\ k_{s} / 100 & \text { when } 2.5 d_{a}<d \leq d_{M} \\ 0 & \text { otherwise }\end{cases}
$$

These rates define a sphere of reach around the walker-anchorage complex, allowing the walker to step onto an uncut anchorage when it is nearby. In Fig. 5 the sphere of reach is depicted to scale with walker circuits. There are two exceptions. Stepping from the initial anchorage and stepping onto the final anchorage occur at lower rates. The 
domain complementary to the walker on the initial anchorage is two bases longer than the corresponding domain of a regular anchorage. Stepping from the initial anchorage was reported to happen $3 \times$ more slowly: this is incorporated in the model. The final anchorage includes a mismatched base that prevents cutting by the nicking enzyme. Based on the experimental data, we fit a tenfold reduction for the rate of stepping onto the final absorbing anchorage (Fig. 4).

Three types of interaction that are known to occur are omitted from the model: all three could be incorporated in future. Firstly, a rate of $k_{s} / 5000$ is reported [15] for transfer of the walker between separate tracks built on different DNA origami tiles. Transfer between tracks could be eliminated by binding the tiles to a surface, thus keeping them apart. Secondly, the walker can move between intact anchorages in the absence of the nicking enzyme with a rate of $\sim k_{s} / 13$ [15]. With the enzyme present, the walker spends little time attached to an intact anchorage, as enzymatic activity is relatively fast. ${ }^{5}$ Therefore we remove the rate altogether. In our model, the anchorage is cut as soon as the walker attaches to it. Thirdly, the walker can step backward onto cut anchorages. This requires a blunt-end strand-displacement reaction which is known to be slow relative to toehold-mediated displacement [17]. A variant of the model with a backward rate $k_{b}=k / 30$ is shown in dotted lines in Fig. 4 (Left). In this case the model predicts significant quenching of fluorophore $\mathrm{F} 2$ at late times by walkers whose forward motion is obstructed by omission of one or more anchorages: this does not match experimental data. A reduced rate $k_{b}=k / 500$ (not plotted) has a similar effect.

The time-dependent responses of fluorescent probes F2 and F8 shown in Fig. 4 (Left) are predicted by the Markov chain model using the rate parameters discussed above without any further fitting: they correspond well to the experimental data.

An additional parameter is needed to model branched tracks (Fig. 5(a)). We introduce a failure rate for the anchorage blocking mechanism which is assumed to be the same for all junctions. We infer a failure rate of $30 \%$ by fitting to the results of the single-layer branched-track experiment illustrated in Fig. 5 [14].

\subsection{Model results}

Having used experiments on straight tracks and with a single layer of branching to determine the parameters of the model, we use the two-layer junction experiments shown in Fig. 5(c) to evaluate its quality. The model captures essential features of the walker behaviour and is reasonably well aligned with experimental data. In the model, not all walkers reach an absorbing anchorage by time $T=200 \mathrm{~min}$, although the predicted quenching is much higher than observed. The reason for this discrepancy is not easily determined and motivates further study.

We exercise the model by model checking them against temporal logic queries aimed at quantifying the reliability and performance of the computation. We note that not all the walkers that finish actually do quench the intended signal. In both the model and the experiments we can identify a difference between paths that follow the side of the track (paths LL and RR), and paths that enter the interior (paths RL and LR):

\footnotetext{
${ }^{5}$ The cutting rate for enzymatic activity was measured at $0.17 \mathrm{~s}^{-1}$, for which the enzyme binding to the DNA is considered not a rate limiting step [3].
} 


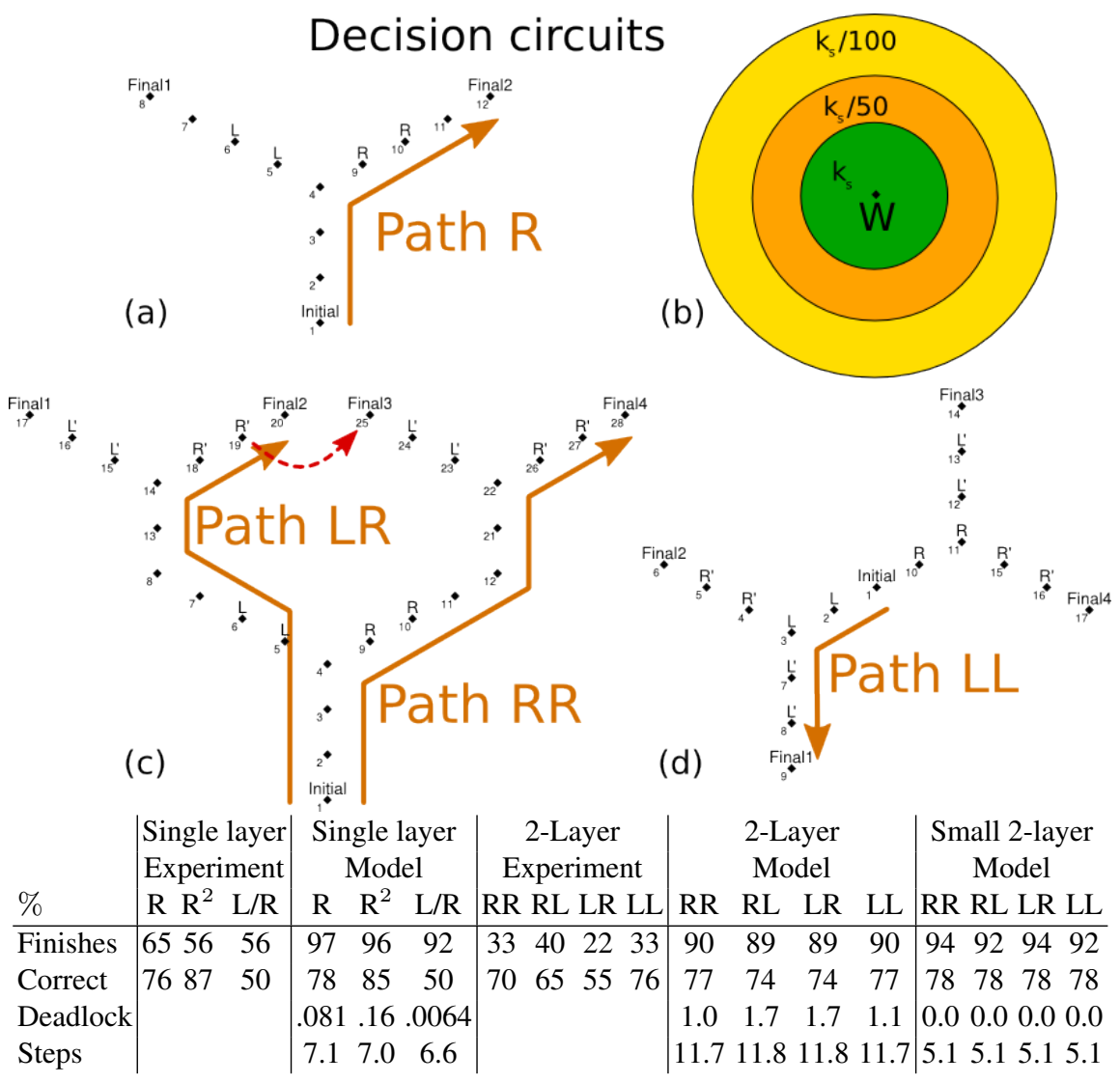

Fig. 5. Top: Track topology for single-layer (a) and double-layer (c,d) decision tracks. Initial indicates the initial anchorage, Final indicates absorbing anchorages, and L, L', R and R' indicate anchorages that can be blocked by input. Coloured circles (b) indicate the range of interaction of the walker to scale. Bottom: Experimental results [14] compared with results from the model. Single layer track: R means a single blockade on the left, $\mathrm{R}^{2}$ means a two-anchorage blockade on the left, L/R means single blockades on both the left and right. Double layer track: RL means anchorages labelled L and R' are blocked, so that the walker goes right on the first decision, and left on the second. Each blockade is of two consecutive anchorages. All properties are given at time $T=200$ min. Finishes, $P_{=?}\left[F^{[T, T]}\right.$ finished $]$, is the probability that a walker quenches any fluorophore by time $T$; Correct, $P=$ ? $\left[F^{[T, T]}\right.$ ("finished-correct"|"finished")], is the probability that a finished walker quenches the correct fluorophore by time $T$; Deadlock, $P_{=?}\left[F^{[T, T]}\right.$ deadlock $]$, is the probability for the walker to get stuck prematurely by time $T$, with no intact anchorage within reach; and Steps, $R_{=\text {? }}$ (steps) $\left[C^{\leq T}\right]$, indicates the expected number of steps taken by time $T$.

the probability of a correct outcome for the side paths is greater. This is explained by leakage transitions between neighbouring paths, for example, see the red dotted line in Fig. 5(d). Walkers on an interior path can leak to both sides, but a path that follows the side can only leak to one side. This effect can also be shown by inspecting paths. By 


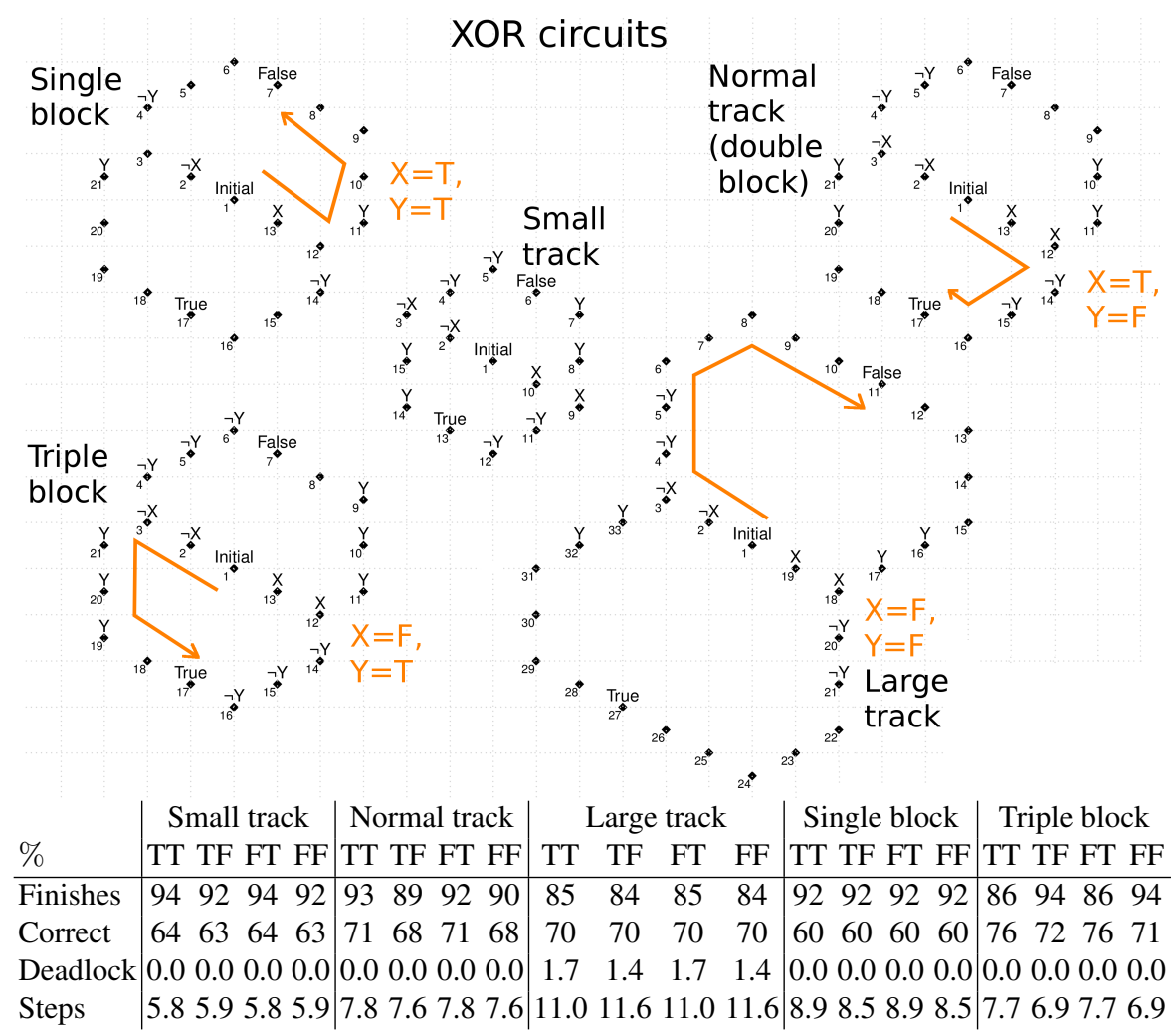

Fig. 6. Performance analysis for a logic track expressing the XOR formula $(X \oplus Y)$. Properties as in Fig. 5.

using the property $P_{=?}$ [ correct-path $U \leq T$ finished-correct ], which denotes the probability that a walker stays on the path until it quenches the correct fluorophore by time $T$, we can reason about the likelihood of the walker deviating from the intended path. For the double-layer track in Fig. 5(d), we infer that the probability of staying on the intended path and reaching the absorbing anchorage within 200 minutes is $55 \%$ for paths LR and RL, and $58 \%$ for paths LL and RR. This shows that walkers on interior paths are indeed more likely to deviate from the intended path than walkers on paths that follow the sides.

The double-layer track can be optimized by reducing the probability of leakage from the intended path. By decreasing the proximity of off-path anchorages and reducing the track length, both the proportion of walkers finishing and correctness are increased (see Fig. 5(d)). The asymmetry between paths (LL, RR vs. LR, RL) also disappears.

Smaller tracks are not always better. In Fig. 6 several variants of a XOR-logic circuit are shown. The 'small', 'normal' and 'large' variants all use a total of four blocker strands per decision node. The large track is approximately as correct as the normal sized track, but a lower proportion of walkers reach an absorbing anchorage. The small 
track has a greater proportion of walkers that finish than the normal sized track, but it is considerably less reliable. We note that the walker has a large range of interaction, which causes leakage and affects the reliability of the computation.

We infer that larger circuits are more susceptible to deadlock, based on Fig. 5 and 6. Deadlock occurs when a walker is isolated on a non-absorbing anchorage with no intact anchorage in range. From a computational standpoint deadlock is undesirable, as it is impossible to differentiate a deadlocked process from a live process.

The performance of PRISM [8] depends on the model checking method. For small tracks as in Fig. 4, verification by PRISM can be achieved using uniformisation with a precision of $10^{-6}$ within $10 \mathrm{~ms}$ on common hardware [1]. Properties for the single layer circuit in Fig. 5 were model checked within 3s to a precision of $<10^{-6}$ using fast adaptive uniformisation [6]. For the dual-layer track in the same figure, single-threaded simulation of $10^{5}$ paths, each of which is checked against the property, yields a $95 \%$ confidence interval of size $<0.4 \%$ within 23 s [1].

\section{Conclusions}

The capability for an autonomous DNA walker to navigate a programmable track has been recently demonstrated [14]. We have considered the potential for this system to implement DNA walker 'circuits'. Working from experimental observations, we have developed a simple model that explains the influence of track architecture, blockade failure and stepping characteristics on the reliability and performance of walker circuits. The model can be further extended as more detailed experimental measurements become available. Model checking enables analysis of path properties and quantitative measures such as the expected number of steps, which cannot be established using traditional ODE frameworks. A major advantage of our approach is that circuit designs can be manipulated to study the properties of variant architectures.

We have shown that walker circuits can be designed to evaluate any Boolean function. In the experimental system we have considered, paths within a circuit can only be joined under specific conditions, resulting in a number of theoretical consequences. One motivation for implementing circuits with a DNA walker system, instead of a DNA strand displacement system (DSD), is the potential for faster reaction times due to spatial locality. However, the walker system we have considered has severely limited potential for parallel circuit evaluation using multiple walkers. As this is not an issue in a DSD, it is the case that this walker system requires exponentially more time to compute certain Boolean functions than a corresponding DSD. This is not necessarily true of all walker systems. The problem arises in the system under consideration due to the undirected nature of the tracks that are traversed by a walker.

Another autonomous walker system with directed tracks has been demonstrated [16] and, in principle, could be extended to have programmable (directed) tracks. In addition to implementing circuits that could be evaluated efficiently by many walkers in parallel, such a system could also benefit from well established design techniques to improve overall circuit reliability [9]. Furthermore, current walker technology 'destroys' the track that is traversed. New mechanisms that can either replenish the track, or can avoid 'destroying' it, will lead to reusable circuits. Finally, it would be interesting to ex- 
plore the information processing capabilities of DNA walkers beyond circuit evaluation and the potential for multiple interacting walkers to exhibit emergent behaviour.

Acknowledgements We thank Masami Hagiya, Jonathan Bath and Alex Lucas for useful discussions. The authors are supported by a Microsoft Research scholarship (FD), ERC AdG VERIWARE, EPSRC Grant EP/G037930/1, a Royal Society - Wolfson Research Merit Award (AJT), and Oxford Martin School.

\section{References}

1. Intel i5-2520M, Fedora 3.8.4-102.fc17.x86_64, OpenJDK RE-1.7, PRISM 4.0.3

2. Bath, J., Green, S.J., Turberfield, A.J.: A free-running DNA motor powered by a nicking enzyme. Angewandte Chemie (International ed. in English) 44(28), 4358-61 (Jul 2005)

3. Bellamy, S.R.W., Milsom, S.E., Scott, D.J., Daniels, L.E., Wilson, G.G., Halford, S.E.: Cleavage of individual DNA strands by the different subunits of the heterodimeric restriction endonuclease BbvCI. Journal of molecular biology 348(3), 641-53 (May 2005)

4. Bryant, R.E.: Symbolic boolean manipulation with ordered binary-decision diagrams. ACM Computing Surveys 24(3), 293-318 (1992)

5. Chandran, H., Gopalkrishnan, N., Phillips, A., Reif, J.: Localized hybridization circuits. DNA Computing and Molecular Programming 6937, 64-83 (2011)

6. Dannenberg, F., Hahn, E.M., Kwiatkowska, M.: Computing cumulative rewards using fast adaptive uniformisation. In: Proc. 11th Conference on Computational Methods in Systems Biology (CMSB'13) (2013), to appear.

7. Green, S., Bath, J., Turberfield, A.: Coordinated chemomechanical cycles: a mechanism for autonomous molecular motion. Physical review letters 101(23), 238101 (2008)

8. Kwiatkowska, M., Norman, G., Parker, D.: PRISM 4.0: Verification of probabilistic real-time systems. Computer Aided Verification 6806, 585-591 (2011)

9. von Neumann, J.: Probabilistic logics and synthesis of reliable organisms from unreliable components. In: Shannon, C., McCarthy, J. (eds.) Automata Studies. pp. 43-98. Princeton University Press (1956)

10. Omabegho, T., Sha, R., Seeman, N.C.: A bipedal DNA Brownian motor with coordinated legs. Science 324(5923), 67-71 (2009)

11. Qian, L., Soloveichik, D., Winfree, E.: Efficient Turing-universal computation with DNA polymers. DNA Computing and Molecular Programming pp. 123-140 (2010)

12. Qian, L., Winfree, E.: Scaling up digital circuit computation with DNA strand displacement cascades. Science 332(6034), 1196-1201 (2011)

13. Seelig, G., Soloveichik, D., Zhang, D., Winfree, E.: Enzyme-free nucleic acid logic circuits. Science 314(5805), 1585-1588 (2006)

14. Wickham, S.F.J., Bath, J., Katsuda, Y., Endo, M., Hidaka, K., Sugiyama, H., Turberfield, A.J.: A DNA-based molecular motor that can navigate a network of tracks. Nature nanotechnology 7(3), 169-73 (Mar 2012)

15. Wickham, S.F.J., Endo, M., Katsuda, Y., Hidaka, K., Bath, J., Sugiyama, H., Turberfield, A.J.: Direct observation of stepwise movement of a synthetic molecular transporter. Nature nanotechnology 6(3), 166-9 (Mar 2011)

16. Yin, P., Yan, H., Daniell, X.G., Turberfield, A.J., Reif, J.H.: A unidirectional DNA walker that moves autonomously along a track. Angewandte Chemie International Edition 43(37), 4906-4911 (2004)

17. Zhang, D.Y., Winfree, E.: Control of DNA strand displacement kinetics using toehold exchange. Journal of the American Chemical Society 131(47), 17303-17314 (Dec 2009) 